\section{The missing link? \\ Sudden infant death syndrome and the diaphragm}

The cause of death in sudden
infant death syndrome (SIDS) is
unknown. Scientists know that
risk factors include the prone
sleeping position, prematurity,
low birth weight, and non-
lethal infections. SIDS likely has
a respiratory origin, but the
diaphragm that powers the vital
respiratory pump has not been
adequately investigated in the
context of SIDS. Diaphragm
failure is a well-known cause of
death in adults, and SIDS-Critical
Diaphragm Failure (SIDS-CDF)
hypothesis posits that the
diaphragmm plays a central role in
SIDS. Pontus Siren investigates
the diaphragm in the context
of SIDS.

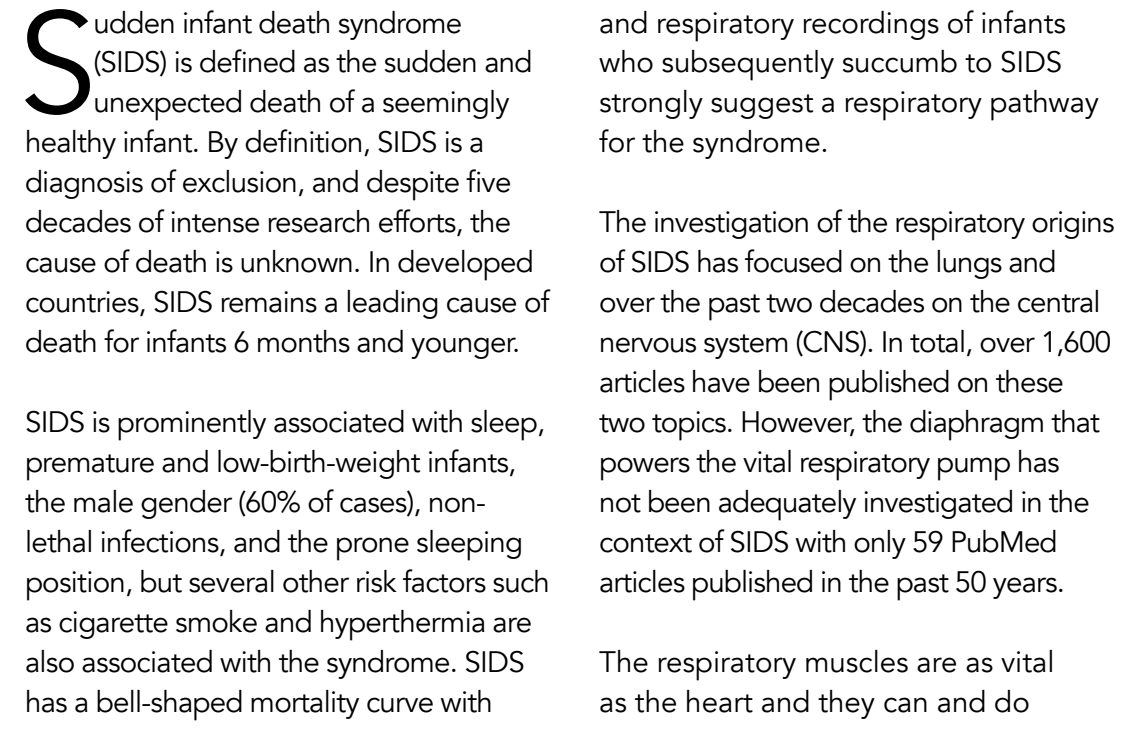

The respiratory muscles (RM) are as vital as the heart and they can and do fail. RM failure is a well known terminal event in adults.

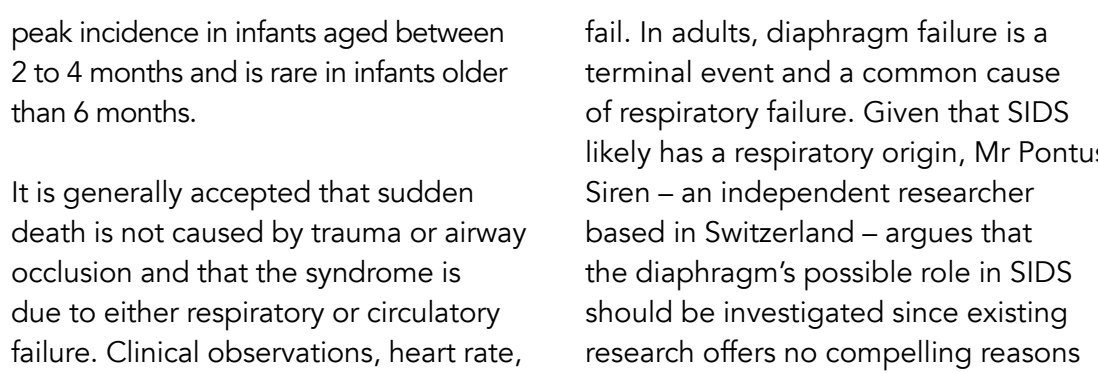

It is generally accepted that sudden death is not caused by trauma or airway due to either respiratory or circulatoy failure. Clinical observations, heart rate,

ail. In adults, diaphragm failure is a terminal event and a common cause likely has a respiratory origin Mr Pontus Siren - an independent researcher based in Switzerland - argues that should be investigated since existing research offers no compelling reasons

Sudden infant death syndrome (SIDS)
is prominently associated with sleep. Q

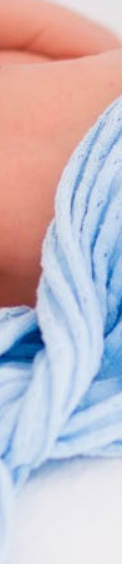

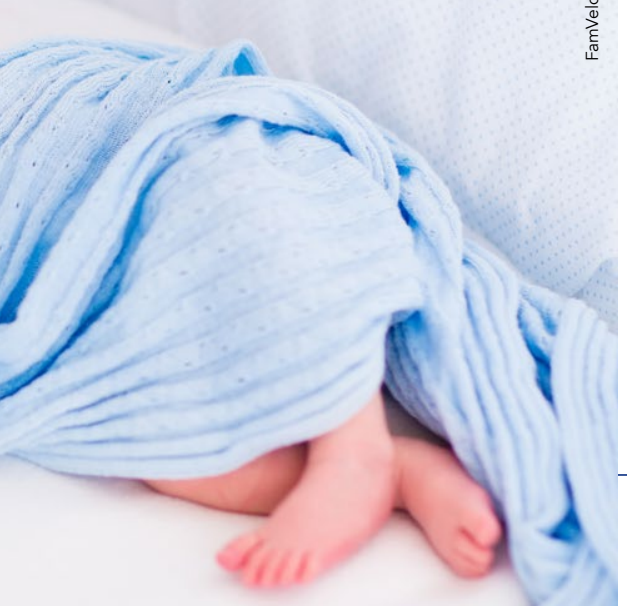
to exclude it.

Mr Siren has written about the diaphragm and SIDS since 2010 and advanced the SIDS-Critical Diaphragm Failure (SIDS-CDF) hypothesis to explain the link between SIDS and the the diaphragm is a vital organ that mus continuously generate adequate force a terminal event and the cause of death IIDS. The hypothesis argues that workload of the diaphragm or reduce its force generating capacity, and that whil SIDS has many conthbuting factors, it vital respiratory pump.

THE ROLE OF THE PRONE SLEEPING POSITION AND NONLETHAL INFECTIONS IN SIDS Research has identified many risk factors for SIDS, but the prone sleeping position and non-lethal infections are especially important. Indeed, a strong correlation and seasonal pattern between SIDS cases and deaths due to respiratory infections has been reported in infants (Figure 1). Similarly, following the "safe sleep" campaigns around the world to encourage infants to sleep on their backs, SIDS

deaths decreased between $40 \%$ and $80 \%$.

Research has shown that even minor

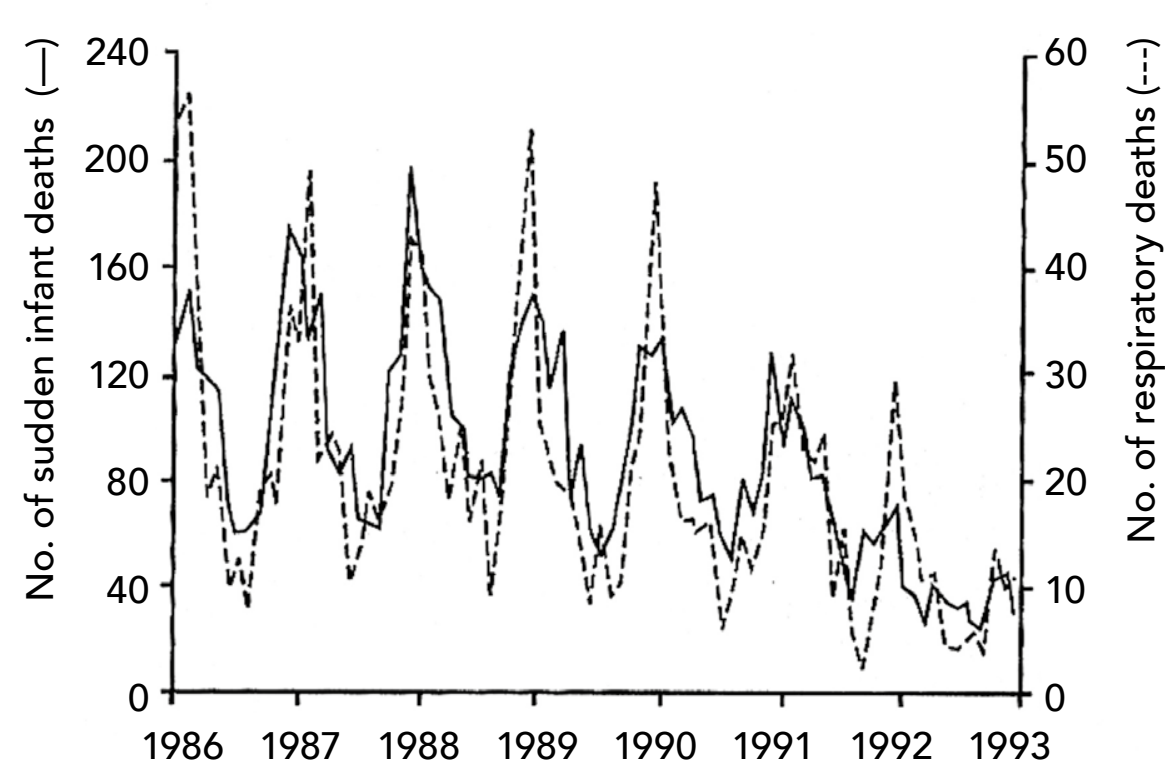

Year

\section{Figure 1. Graph illustrates the strong correlation and seasonal pattern between SIDS cases
and deaths due to respiratory infections in infants. Reproduced with permission from BMJ
1995:310:1603 htsed}

Research has shown that even minor infections can significantly reduce the force generating capacity of the human diaphragm. In experimental settings, infections have been shown to reduce the diaphragm force generating capacity by as much as $\mathbf{5 0 \%}$ in $\mathbf{2 4}$ hours.

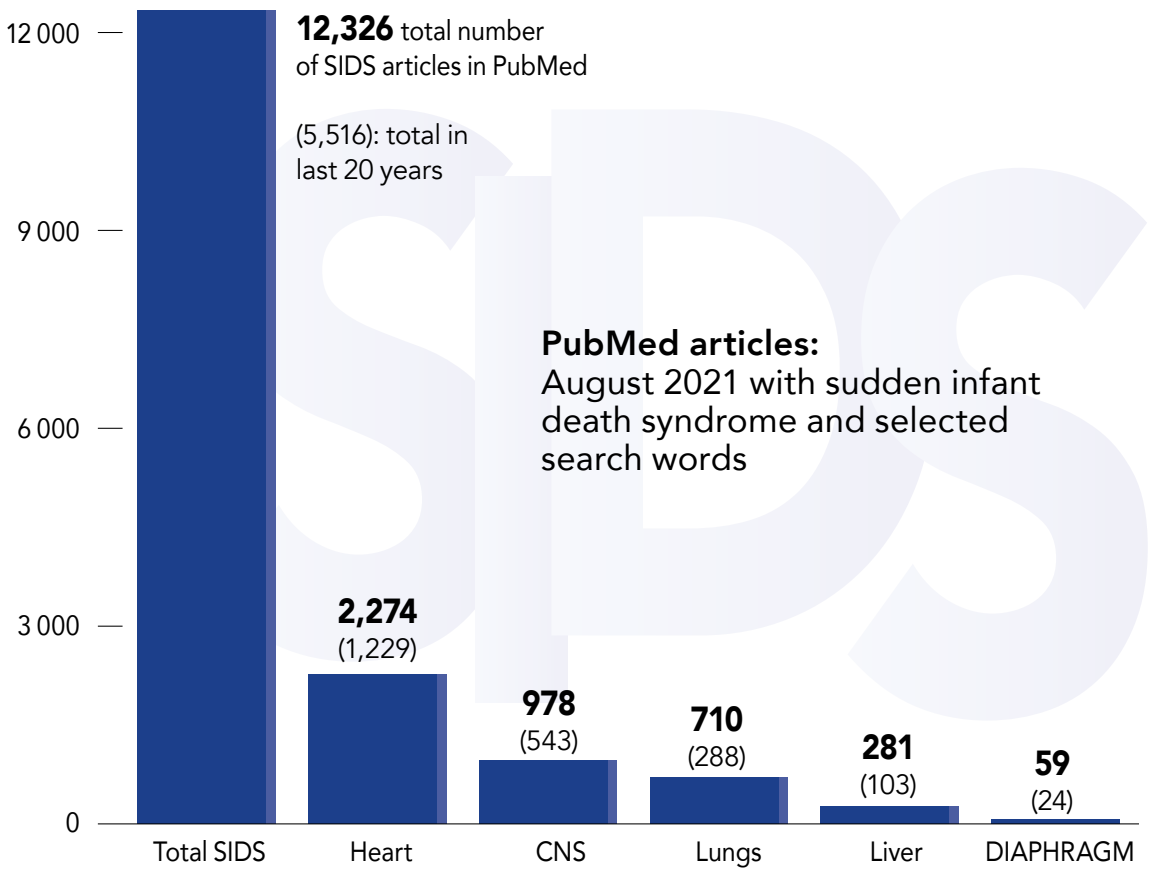
diaphragm. The hypothesis posits that

Figure 2. The diaphragm's possible role in SIDS has not been adequately investigated. force generating capacity of the human diaphragm and in experimental settings, infections have been shown to reduce by as much as $50 \%$ in 24 hours. In adult patients, routine in fections have been shown to result in shortness of breath, reduction in vital capacity, and acute that in vulnerable populations, even minor infections can induce a significant reduction in the diaphragm's force generating capacity.

The one pathological finding that characterises over $70 \%$ of SIDS cases is of small red or purple spots caused by bleeding into the intrathoracic tissue. Research shows that an infection together with gasping or a "struggle to breathe" episode is required for the formation of characteristic intrathoracic petechia haemorrhages. The clinical signs of respiratory muscle fatigue are rapid movements, and respiratory pauses combined with hyperventilation.

Currently there is no commonly accepted mechanism to explain why the prone sleeping position is such an important risk factor in SIDS. Mr Siren points out that the prone sleeping position significantly increases the compared to the supine position. He hypercapnia. Research further shows shallow breathing, paradoxic chest wall 


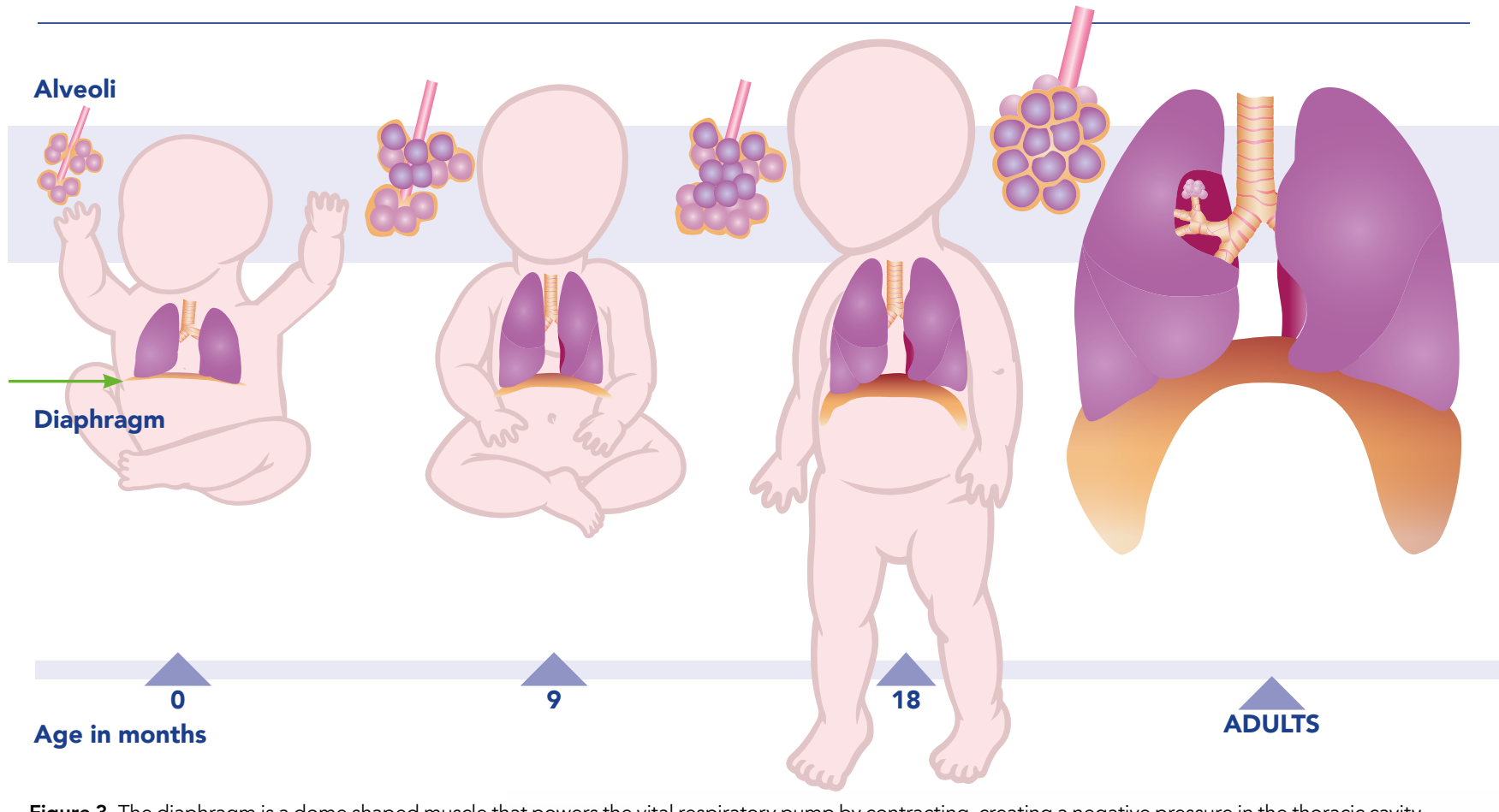

Figure 3. The diaphragm is a dome shaped muscle that powers the vital respiratory pump by contracting, creating a negative pressure in the thoracic cavity,
and drawing air into the lungs. The diaphragm develops rapidly during the first 18 month of of life and reaches adult levels of Pdi Pd $_{\text {max }}$ at approx 6 months of age.

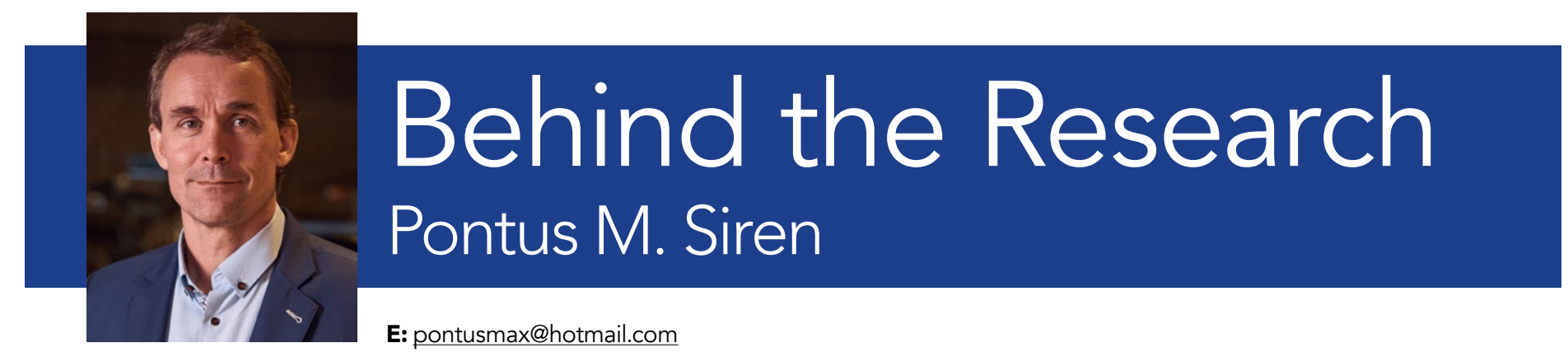

can be established. Of the critical role of the heart, lungs, and central nervous system in SIDS has been investigations have not produced a satisfactory explanation of the there is a broad consensus that SIDS likely has a respiratory origin. Mr Siren has repeatedly argued that the diaphragm, which powers the critical respiratory pump,

$\begin{array}{cc}\text { The diaphragm is the most important } & \begin{array}{l}\text { remains an serious } \\ \text { blind spot in SIDS } \\ \text { muscle in the human body after the }\end{array}\end{array}$

$\begin{array}{cl}\text { The diaphragm is the most important } & \begin{array}{l}\text { remains an serious } \\ \text { blind spot in SIDS }\end{array} \\ \text { muscle in the human body after the } & \text { research. }\end{array}$ heart, and in pre-term infants is only as MrSiren suggests thick as 10-17 sheets of paper. $\quad$ is needed to explore

diaphragm fatigue and, in conjunction the diaphragm in SIDS. He highlights with other risk factors, lead to critical diaphragm failure. the imperative to investigate the link and SIDS, and proposes that scientists leverage research on the adult animals and humans have explored how diaphragm and its dysfunctions in the cigarette smoke contributes to SIDS. The context of SIDS

findings conclude that cigarette smoke can affect the energy metabolism of the diaphragm, leading to reduced force generating capacity

\section{THE MISSING LINK?}

By definition, SIDS is a diagnosis of

Mr Siren hopes that by highlighting the possible role of the diaphragm in SIDS, researchers will start and progress towards understanding the underlying mechanism of cardiorespiratory organs, the possible extensively investigated. These origins of the syndrome. However, investigating the missing link in SIDS

\section{Research Objectives}

Pontus Siren highlights the fact that the diaphragm has not been adequately investigated in the context of sudden infant death syndrome (SIDS) and that it may be the missing link in the syndrome.

\section{Detail}

Bio

Pontus Siren is an independent researcher based in Switzerland
References

Siren, PM (2021). Blind spot. Pediatr. Res, https://doi.org/10.1038/s41390-021-01508-4

Siren, PM (2017). SIDS-CDF Hypothesis Revisited: Cause vs. Contributing Factors. Front. Neurol., 7, 244 https://doi.org/10.3389/fneur.2016.00244

Siren, PM \& Siren, MJ (2011). Critical diaphragm failure in 115-123. htps:/doiorg/103109/030097342010.548011

\section{Personal Response}

How do you think scientists could be encouraged and supported to undertake more research projects looking

II SIDS has been intensively investigated for over five decades, but the cause of death has not critical cardiorespiratory organs have been comprehensively investigated in the context of SIDS. The diaphragm has been extensively studied in other vulnerable patient populations and this large body of evidence should be leveraged in SIDS research. The respiratory pump is as vital as the SIDS research. 